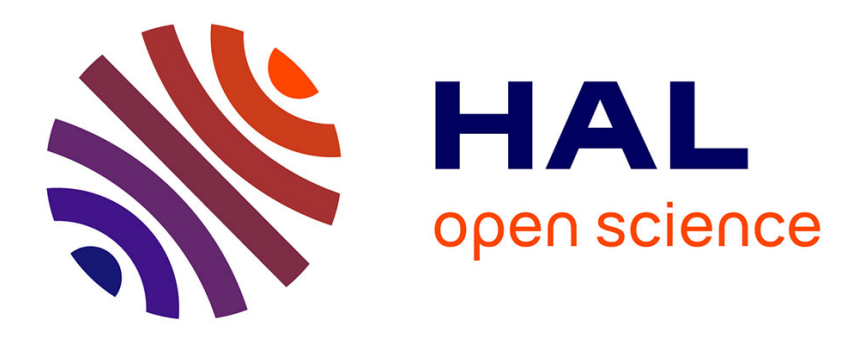

\title{
Approche historique des classifications en psychiatrie
}

\author{
J. Garrabé
}

\section{To cite this version:}

J. Garrabé. Approche historique des classifications en psychiatrie. Annales Médico-Psychologiques, Revue Psychiatrique, 2011, 10.1016/j.amp.2011.03.002 . hal-00748148

\section{HAL Id: hal-00748148 \\ https://hal.science/hal-00748148}

Submitted on 5 Nov 2012

HAL is a multi-disciplinary open access archive for the deposit and dissemination of scientific research documents, whether they are published or not. The documents may come from teaching and research institutions in France or abroad, or from public or private research centers.
L'archive ouverte pluridisciplinaire HAL, est destinée au dépôt et à la diffusion de documents scientifiques de niveau recherche, publiés ou non, émanant des établissements d'enseignement et de recherche français ou étrangers, des laboratoires publics ou privés. 


\section{Accepted Manuscript}

Title: Approche historique des classifications en psychiatrie

Author: J. Garrabé

PII:

S0003-4487(11)00067-9

DOI: doi:10.1016/j.amp.2011.03.002

Reference: $\quad$ AMEPSY 1311

To appear in: $\quad$ Annales Médico-Psychologiques

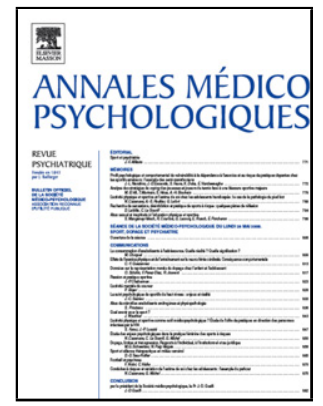

Please cite this article as: Garrabé $\mathrm{J}$, Approche historique des classifications en psychiatrie, Annales medio-psychologiques (2010), doi:10.1016/j.amp.2011.03.002

This is a PDF file of an unedited manuscript that has been accepted for publication. As a service to our customers we are providing this early version of the manuscript. The manuscript will undergo copyediting, typesetting, and review of the resulting proof before it is published in its final form. Please note that during the production process errors may be discovered which could affect the content, and all legal disclaimers that apply to the journal pertain. 


\title{
Communication
}

\section{Approche historique des classifications en psychiatrie An historical approach of classifications in psychiatry J. Garrabé}

\author{
7, place Pinel, 75013 Paris, France
}

Auteur correspondant : Docteur Jean Garrabé, 7, place Pinel, 75013 Paris, France

Adresse email : jean.garrabe@wanadoo.fr

\section{Résumé}

Dès le milieu du $\mathrm{XIX}^{\mathrm{e}}$ siècle s'est posée la question des critères de classification des maladies. Pour les maladies mentales, diverses classifications ont alors été proposées par des auteurs français (Morel) et allemands (Kahlbaum, Kraepelin). À partir de la fin du XIX ${ }^{\mathrm{e}}$ siècle, le Bureau International de Statistique (Paris) a publié à une Classification Internationale des Maladies, à révision décennale (J. Bertillon). Cette tâche a été poursuivie dans l'entre-deuxguerres par le Bureau de Santé de la Société des Nations (Genève) puis, après la Seconde Guerre mondiale, par l'OMS à partir de la $5^{\mathrm{e}}$ édition. Un chapitre spécial V $(\mathrm{F})$ a ensuite été réservé aux troubles mentaux. Plusieurs classifications nationales ont été révisées et modifiées pour établir des concordances avec les successives éditions de la CIM, et en particulier, dans le domaine de la psychiatrie infanto-juvénile, la Classification Française des Troubles Mentaux de l'Enfant et de l'Adolescent dans sa version 2010.

Mots clés : Classification des maladies ; Psychiatrie infanto-juvénile

\begin{abstract}
The question of finding criteria to help classify diseases started arising from the middle of the 19th century onwards. As for mental diseases French (Morel) and German (Kahlbaum, Kraepelin) authors proposed several classifications. From the end of the $19^{\text {th }}$ century the International Bureau of Statistics (Paris) started publishing an International Classification of Diseases, to be reviewed every ten years (J. Bertillon). The Health Bureau of the League of Nations (Geneva) continued this work in the years between the two world wars, then the WHO carried on after the second world war for the $5^{\text {th }}$ edition onwards. A specific
\end{abstract}


chapter V (F) was devoted to mental illness. Several national classifications have been revised and modified to establish a concordance with the successive editions of the ICD especially, regarding the area of child psychiatry, the French Classification of Child and Adolescent Mental Diseases in the 2010 version.

Keywords: Child psychiatry; Disease classification

La question de savoir quelles classifications des troubles mentaux utiliser est présente tout au long de l'histoire de la psychiatrie. Dès la période de la psychopathologie descriptive [2], de la fin du XIX siècle au début du XX ${ }^{\mathrm{e}}$, les deux écoles alors actives dans la description d'entités nosologiques nouvelles définies par leur symptomatologie, la française et l'allemande, ont proposé de les classer différemment selon que les symptômes retenus sont ceux observés en clinique ou ceux évocateurs d'une étiologie supposée. Une des plus célèbres en France de ces classifications est celle « des maladies mentales dans leurs rapports avec les relations intimes nécessaires, qui existent entre la forme de la folie et la nature de la cause » proposée en 1860 par B.-A. Morel [17]. La Société Médico-Psychologique ne consacra pas moins de neuf séances à sa discussion, dont les comptes rendus furent publiés dans les Annales Médico-Psychologiques au cours de l'année 1861. Jules Falret y fit le 26 décembre 1860 un discours sur «Les principes à suivre dans les classifications des maladies mentales », qu'il mit en tête des «Études cliniques sur les maladies mentales et nerveuses » publiées en 1890 alors qu'il la présidait [5].

En Prusse, K.-L. Kahlbaum proposa en 1863 une classification incluant une forme particulière de «folie de la jeunesse », l'hébéphrénie, dont les symptômes apparaissent à la puberté [10].

En 2002, notre société a consacré une séance à la présentation de la CFTMEA R.2000 [15], au cours de laquelle j'ai moi-même parlé de la dimension culturelle des classifications en psychiatrie [7].

À la fin du XIX ${ }^{\mathrm{e}}$ siècle était apparue la nécessité, pour des raisons avant tout démographiques, de recueillir des données concernant les maladies contagieuses ou épidémiques permettant d'établir des statistiques de mortalité dans les populations atteintes. Le Bureau International de Statistique installé à Paris utilisa pour ce faire le système conçu par Jacques Bertillon, médecin, démographe, qui entreprit d'en publier des révisions décennales qu'il effectuait seul. Ce «Classement des causes de la mort » fut ensuite complété 
par une «Classification des maladies » susceptibles de l'entraîner, où la pathologie mentale n'était représentée que par le suicide, l'alcoolisme et la paralysie générale. Dans l'entre-deuxguerres, un bureau spécialisé de la Société des Nations à Genève prit le relais de Bertillon, effectuant la Cinquième révision décennale (1938); les grands pays qui ne faisaient pas partie de la SDN ne participaient pas à ces travaux. En France fut proposée en 1936 une réforme de la «Statistique des aliénés» avec un système à double entrée « Nomenclature » et «Étiologie » qui ne put être mis en œuvre en raison du déclenchement de la Seconde Guerre mondiale.

Pendant la première moitié $\mathrm{du} \mathrm{XX}^{\mathrm{e}}$ siècle coexistèrent donc en psychiatrie plusieurs classifications dites «modernes » par Henri Ey dans l'Étude psychiatrique $\mathrm{n}^{\circ} 20$ consacrée à leur analyse : celle de Kraepelin dans sa version de 1906 qui distingue XV groupes de maladies, la classification «classique» de l'École française qui en retient XII, et celle proposée en 1934 par l'APA qui en retenait XVIII [4]. Dans les trois ne figure pour la psychiatrie de l'enfant qu'une rubrique, «Arriérations ou Arrêts de développement psychique», celle de l'APA distinguant cependant les «psychoses avec arriération intellectuelle ». Le Premier Congrès Mondial de Psychiatrie de Paris en 1950 a montré que les échanges internationaux étaient rendus difficiles par l'emploi de terminologies différentes, certains termes semblables en apparence ne désignant pas les mêmes concepts dans chaque école. L'Association Mondiale de Psychiatrie créa une section «Classification» pour comparer et harmoniser celles existantes.

De son côté, l'OMS, créée par l'ONU après la Seconde Guerre mondiale, ayant pris le relais du bureau de la SDN, fit paraître en 1948 la sixième mise à jour de la classification Bertillon désormais intitulée Classification Internationale des maladies, traumatismes et causes des décès, la première publiée par cette agence. La CIM-6 (ICD6) cessait de répertorier seulement les seules causes de décès pour s’intéresser de façon plus générale à la morbidité, la pathologie mentale ne faisant cependant pas l'objet d'un chapitre spécifique.

La CIM est une classification statistique et monoaxiale. Statistique en ce sens qu'un code correspondant à une entité répertoriée ne peut être attribué qu'à une et une seule catégorie de la classification, d'où des règles de codage pour le choix de l'affection principale. Monoaxiale en ce que chaque entité ne correspond qu'à un seul code avec des règles d'exclusion.

Un auteur comme H.-F. Ellenberger avertit les «psychiatres classificateurs » des dangers qui les guettent aussi bien s'ils proposent une classification «naturelle» ou «artificielle », ou même s'ils déclarent que l'on ne peut classer les maladies mentales [3], ce 
qui paradoxalement est une manière de les classifier comme inclassables. Au moment de la vogue de l'antipsychiatrie, l'intérêt pour la nosographie a disparu un temps, désintérêt qui se produisit aussi chez ceux qui pensent que l'on ne pourra classer les maladies mentales que lorsque seront connus les mécanismes bio-cérébraux à leur origine. Ellenberger signale une communication faite par des auteurs péruviens au troisième Congrès Mondial de Psychiatrie de Montréal en 1961 sur l'intérêt de l'analyse statistique de la fréquence des symptômes pour déterminer des catégories diagnostiques, annonce de l'abandon des critères psychopathologiques au profit du calcul mathématique pour déterminer les critères retenus comme significatifs d'une catégorie diagnostique.

La publication en 1968 par la section psychiatrie de l'INSERM d'une Classification française des troubles mentaux en 20 catégories, rédigée en collaboration avec le CCOMS de Paris, passa inaperçue en raison de la date de parution de ce texte. Elle reste proche de celle dite «classique» et concerne la psychiatrie générale mais on peut noter qu'en 02 Schizophrénie chronique est classée 02.7 Schizophrénie chronique, forme de l'enfant et 02.8 Autisme infantile et autres formes de psychose infantile précoce [9].

La septième édition de la CIM n'apporte pas de grandes nouveautés dans notre domaine ; en revanche, la huitième révision (1974) s'accompagne d'un Glossaire et guide de classification des troubles mentaux à utiliser en liaison avec le chapitre V (Troubles mentaux). Elle était précédée de l'avant-propos rédigé par sir Aubrey Lewis pour la septième, dont il avait supervisé la rédaction où il précisait que «les troubles énumérés dans ce glossaire sont identifiés à l'aide de critères essentiellement descriptifs », c'est donc toujours une psychopathologie descriptive qui reste la référence de ces deux révisions. Si ce chapitre V (F) marque que la pathologie mentale occupe une place particulière dans la CIM, dans d'autres chapitres figurent des maladies physiques qui, lorsqu'elles existent chez un malade mental, doivent aussi être codées. En ce qui concerne la psychiatrie infantile, il faudra repérer par un second code des maladies ou des facteurs pathogènes propres à l'enfance figurant dans d'autres chapitres de la CIM. Il y a d'ailleurs une différence radicale entre un chapitre traitant des troubles mentaux faisant partie d'une classification générale des maladies, comme la CIM, et une classification isolée des Troubles mentaux isolée comme le DSM.

La neuvième révision du chapitre V de la CIM (1979) est l'aboutissement d'une série de colloques organisés par l'OMS, dont un à Paris en 1967, consacré aux Troubles psychiatriques de l'enfance, au cours duquel R. Misès souligna à propos des Problèmes nosologiques posés par les psychoses de l'enfant [16] que leur classification doit tenir compte non seulement de la symptomatologie, mais aussi des éléments d'une psychopathologie 
structurale du fonctionnement mental. Un autre aspect novateur de cette révision est que pour la première fois est envisagée l'élaboration d'une classification spécifique des troubles mentaux de l'enfant et de l'adolescent.

Une «liste abrégée » donne les nouvelles rubriques spécifiques pour les troubles de l'enfance et de l'adolescence prévues dans la CIM-9 [19] :

\section{Psychoses spécifiques de l'enfance.}

299.0. Autisme infantile.

299.1. Psychose désintégrative.

299.8. Autres.

299.9. Sans précision.

\section{Troubles de l'activité spécifique de l'enfance et de l'adolescence.}

\section{Syndrome d'instabilité de l'enfance.}

\section{Retards spécifiques du développement.}

Lors du sixième Congrès Mondial d'Honolulu en 1977, la Section classification a adopté une résolution invitant les sociétés nationales de psychiatrie qui possédaient une classification à les réviser pour les rendre compatibles avec la dixième révision de la CIM. Le résultat paradoxal fut que l'APA procédant elle-même à la révision du DSM-II prit de vitesse les classificateurs de l'OMS en publiant dès 1980 la troisième édition qui effectuait une véritable révolution taxinomique [1]. Le DSM-III connut à travers ses traductions un succès mondial avec le résultat inattendu. Alors que ce qui était souhaité était que les classifications nationales se mettent en concordance avec la CIM, c'est l'inverse qui se produisit. Plusieurs auteurs français s'interrogèrent alors sur l'application du DSM-III à la psychiatrie de l'enfant et de l'adolescent [12].

Les travaux pour élaborer la CIM-10 ont été entrepris à l'OMS en 1983. Dans un numéro «Classification et psychiatrie » de Confrontations psychiatriques, des représentants de plusieurs pays et institutions, dont l'OMS, faisaient en 1984 le point sur des problèmes théoriques. La question des classifications était abordée pour d'autres âges de la vie que l'enfance et l'adolescence, comme la psychiatrie gériatrique, ou selon d'autres points de vue, comme ceux de la psychanalyse, de la génétique ou de la nosologie. L. Kreisler abordait les Problèmes spécifiques de classification en psychiatrie dans le premier âge. Propositions nosographiques nouvelles [11]. R. Misès et Ph. Jeammet, à propos de La nosographie en psychiatrie de l'enfant et de l'adolescent, sans reprendre l'étude historique du problème, 
renvoyant sur ce point à un texte antérieur de M. Rutter [18], et après avoir souligné que CIM-9 et DSM-III participent de deux démarches très différentes, annoncent la mise en place d'un groupe de travail pour concevoir une classification des troubles mentaux spécifiques de l'enfance et de l'adolescence [14]. Ce sera la CFTMEA qui établit des correspondances avec l'édition concordante de la CIM ; la quatrième édition CFTMEA-R-2000 reproduit, avec l'autorisation de l'OMS, le chapitre V (F) de la CIM-10 (1992).

Nous avons tenté en 1989 d'établir uniquement pour ce qui est de la psychiatrie générale des correspondantes entre les catégories diagnostiques retenues par les différentes classifications alors en usage, INSERM, DSM-III, DSM-III-R, projet de révision de la CIMIX [6]. Mais nous avons eu le sentiment de gravir une véritable tour de Babel, les classifications proposées n'ayant pas le même objet, d'où l'erreur d'utiliser celles conçues pour recueillir des données statistiques sur des grandes cohortes de malades comme outil d'investigation psychopathologique dans l'analyse clinique individuelle.

Lors des $28^{\text {es }}$ journées de la SIP «Psychoses: évolution des pratiques et des concepts », ont été traités l'histoire du concept de «psychose de l'enfant » [8] et le problème de la classification de l'autisme et des psychoses précoces avec un article de R. Misès, N. Garret-Gloanec et Y. Coinçon, où figurent des tableaux de correspondance entre la CFTMEA-R-2000 et la CIM-10 [13].

Nous ne pensons pas que la parution du DSM-IV en 1994 ait permis de rattraper le décalage qui est plus conceptuel que temporel. Pour le moment est annoncée une révision à minima de la CIM-10, la CIM-11 ne devrait pas connaître un grand bouleversement, ni son alignement pur et simple sur le DSM-V actuellement en discussion puisque la parution de ce dernier est reportée à 2013 en raison de la difficulté à obtenir un accord au sein des groupes de travail pour certaines des catégories diagnostiques retenues en psychiatrie générale.

J'ajouterai pour conclure que des révisions trop fréquentes ou rapprochées des classifications en psychiatrie, quel que soit leur objectif, paraissent aller à l'encontre du but recherché. Des changements dans la définition des catégories peuvent augmenter la prévalence apparente d'un trouble ou fausser l'évaluation au long cours des résultats obtenus par telle ou telle méthode thérapeutique utilisée pour le traiter.

Il est utile de rappeler cet historique des classifications internationales des maladies au moment où se tiennent aussi bien à Genève qu'au COOMS de Lille des réunions auxquelles participent non seulement des psychiatres, mais aussi des représentants des autorités sanitaires et des associations de malades ou de familles de malades pour se prononcer sur les modifications à apporter à la CIM-11 pour le chapitre $\mathrm{V}(\mathrm{F})$, avec des changements de 
dénomination de plusieurs catégories diagnostiques, question déjà évoquée au cours de séances de l'année 2010 de notre Société.

\section{Conflit d'intérêt : à compléter par l'auteur}

\section{Références}

[1] American psychiatric Association. DSM-III ; Manuel diagnostique et statistique des troubles mentaux. Traduction française. Paris: Masson; 1983.

[2] Berrios G. The history of mental symptoms. Descriptive psychopathology since the nineteenh. Century. Cambridge Université Press; 1996.

[3] Ellenberger HF. Les illusions des classifications psychiatriques. Évolution psychiatrique $1963 ; 28: 221-42$.

[4] Ey H. Études psychiatriques. Étude $n^{\circ} 20$. La classification des maladies mentales et le problème des psychoses aiguës. Paris: Desclée de Brouwer; 1954.

[5] Falret J. Principes à suivre dans les classifications des malades mentales (discours prononcé à la Société Médico-Psychologique le 26 novembre 1860). In: Études cliniques sur les maladies mentales et nerveuses. Paris: Baillière et fils; 1890. p.1-31.

[6] Garrabé J. Dictionnaire taxinomique de psychiatrie. Paris: Masson; 1989.

[7] Garrabé J. La dimension culturelle des classifications des maladies mentales. Ann Méd Psychol 2002;3:253-6.

[8] Hochmann J. Histoire et actualité du concept de psychose de l'enfant. L'Information psychiatrique, 2010;86:227-35.

[9] Institut national de la santé et de la Recherche médicale. Classification française de troubles mentaux. Paris: 1968.

[10] Kahlbaum KL. Die Gruppering der psychischen Krankheiten und die Eintheilung der Seelenstorungen. Danzig: A.W. Kafemann; 1863.

[11] Kreisler L. Problèmes spécifiques de classification en psychiatrie dans le premier âge. Propositions nosographiques nouvelles. In: Confrontations psychiatriques. $\mathrm{N}^{\circ} 24$. Classification et psychiatrie. Paris: Specia; 1984.

[12] Lefèvre $\mathrm{M}$, Lebovici $\mathrm{S}$, Jeammet $\mathrm{Ph}$. L'application de la nouvelle classification américaine dite DSM-III à la psychiatrie de l'enfant et de l'adolescent. Psychiatrie de l'enfant 1968;11:493-512.

[13] Misès R, Garret-Gloannec N, Coinçon Y. Classification de l'autisme et des psychoses infantiles, plaidoyer pour des convergences. L'Information psychiatrique, 2010;86:223-6. 
[14] Misès R, Jeammet Ph. La nosographie en psychiatrie de l'enfant et de l'adolescent. In: Confrontations psychiatriques. № 24. Classification et psychiatrie. Paris: Specia; 1984.

[15] Misès R, Quémada N. Classification Française des Troubles mentaux de l'enfant et de l'adolescent-R-2000. Quatrième édition. Paris: CTNERHI; 2002.

[16] Misès R. Problèmes nosologiques posés par les psychoses de l'enfant. La psychiatrie de l'enfant 1968;26:493-505.

[17] Morel BA. Traité des maladies mentales. Paris: Librairie Victor Masson; 1860.

[18] Rutter M. La classification en psychiatrie infantile. La psychiatrie de l'enfant 1968;11:550-70.

[19] Troubles mentaux : Glossaire et guide de concordance avec la Neuvième Révision de la Classification internationale des maladies.

\section{Discussion}

Dr R. Ropert - Merci à Jean Garrabé, qui vient de nous montrer l'importance des essais successifs de classification dans l'Histoire et la tradition médicale française. Il nous rappelle aussi qu'en présence de certaines de ces variantes classificatoires, il était nécessaire, en fin de compte, de pouvoir les situer par rapport à quelques « racines » fondamentales. En me rendant cet été au Muséum d'Histoire naturelle, j'ai pu constater qu'en matière de classification botanique, on découvrait certaines «variations » récentes, mais qu'en fin de compte, on conseillait toujours de se rapporter à la classification de Linné...

Dr P. Van Amerongen - Je voudrais remercier Jean Garrabé pour sa référence, à mon sujet, aux associations de familles de malades. La question du handicap chez l'enfant impose une classification des classifications des maladies de l'enfant afin d'aider les familles et l'entourage à tenter de comprendre.

$\operatorname{Pr}$ L. Crocq - Je voudrais attirer l'attention sur les erreurs de logique que présentent certaines classifications. Aussi, dans la $8^{\mathrm{e}}$ classification des maladies mentales, après l'énumération des différents diagnostics répartis dans les quatre grandes classes: 1) arriérations, 2) psychoses, 3) névroses et 4) troubles caractériels, voyait-on apparaître de nombreux diagnostics déjà classés dans ces quatre classes, par exemple «troubles mentaux de l'alcoolisme » ou «troubles mentaux de la femme enceinte ». Ainsi, on recoupait le critère sémiologique par un critère étio-pathogénique, pour introduire de nouveaux diagnostics en 
doublon. De la même façon, concernant la classification des troubles mentaux de l'enfant, voyait-on apparaître, en fin d'énumération, des diagnostics désignant un symptôme isolé, sans son contexte sémiologique, par exemple «tics isolés » ou «énurésie isolée ».

Pour mieux me faire comprendre, permettez-moi d'évoquer ma classification des chiens qui admettrait de tels doublons. Je classe les chiens par races, et, quand j'ai fini, je rajoute de nouvelles races de chiens : les chiens mouillés pour être sortis sous la pluie ou tombés dans la rivière, et les chiens dont on n'aperçoit que le bout de l'oreille ou le bout de la queue ; c'est-à-dire introduire impromptu, en concurrence avec le critère descriptif, le critère étio-pathogénique se référant à la cause, et le critère partiellement descriptif, qui se contente d'isoler un signe apparent, et de l'ériger au rang d'entité diagnostique, sans se donner la peine d'inventorier les autres signes présents et de rattacher le tout à un diagnostic cohérent. Il ne faut pas confondre le symptôme considéré isolément, et l'entité diagnostique qui tient compte de ce symptôme en liaison avec les autres symptômes coexistants. 